$\S=$

\title{
Analytical treatment for the conformable space-time fractional Benney-Luke equation via two reliable methods
}

\author{
Khalid K. Ali ${ }^{1}$ *, R.I. Nuruddeen ${ }^{2}$ \\ ${ }^{1}$ Mathematics Department, Faculty of Science, Al-Azhar University, Nasr-City, Cairo, Egypt \\ ${ }^{2}$ Department of Mathematics, Faculty of Science, Federal University Dutse, Jigawa State, Nigeria \\ *Corresponding author E-mail: rahmatullah.n@fud.edu.ng
}

\begin{abstract}
In this study, with help of the Mathematica software, we employ the Kudryashov method and the modified extended tanh expansion method with the Riccati differential equation to analytically treat the Benney-Luke equation. The Benney-Luke equation considered in this study features fractional derivatives in both the spatial and the temporal variables of the newly introduced conformable fractional derivative. We extensively examine the equation via the two methods, and we construct various structures such as the exponential functions, trigonometric functions and hyperbolic functions. Finally, we depict the graphs of all solutions.
\end{abstract}

Keywords: Conformable Fractional Derivative; Space-Time Fractional Benney-Luke Equation; Singular Solution.

\section{Introduction}

Nonlinear partial differential equations featuring fractional order derivatives play important roles in modeling many real-life applications. The study of such equations becomes imperative since they best describe physical situations in many instances. In particular, one can find equations such as the Boussinesq-Burgers equation [1] and Benney-Luke equation [2] to be of paramoun importance in the study of shallow water, fluid dynamics and plasma physics among others. More recently, the classical Benney-Luke equation Eq. (1)

$u_{t t}-u_{x x}+p u_{x x x}-q u_{x x t}+u_{t} u_{x x}+u_{x} u_{x t}=0, x \in[0,1]$

Where $p-q=w-1 / 3$, and $w$ is the surface tension parameter; was studied by Islam et al. in [3] by employing the $G^{\prime} / G$ expansion method. Moreover, one can find many other analytical methods in the literature to study such class of equations, see [420].

On the other hand, the theory of the fractional calculus is gaining weight with various definition ranging from the RiemannLiouville fractional derivative and Caputo's fractional derivative definition [21-22]; and the recent conformable and AtanganaBaleanu fractional derivative definitions [23-24], [25], respectively.

Further, for the conformable fractional derivative; for

$u:[0, \infty) \rightarrow \mathbb{R}$, the $\alpha^{\prime} \mathrm{s}$ order conformable derivative of $u$ is defined by

$D_{t}^{\alpha}(u(t))=\lim _{\varepsilon \rightarrow 0} \frac{u\left(t+\varepsilon t^{1-\alpha}\right)-u(t)}{\varepsilon}, t>0, \alpha \in(0,1]$.

However, in this study, the space-time fractional Benney-Luke equation Eq. (3)
$D_{t t}^{2 \alpha} u-D_{x x}^{2 \alpha} u+p D_{x x x}^{4 \alpha} u-q D_{x x t}^{4 \alpha} u+3 D_{x x t}^{3 \alpha} u^{2}=0, \quad \alpha \in(0,1]$

Will be examined using the newly introduced conformable fractional derivative definition [23-24] by employing two promising and reliable analytical methods. The methods involve are the Kudryashov method [4] and the modified extended tanh expansion method with the Riccati differential equation [5]. The Mathematica software will be fully utilized in the solution aspect as well as in the graphical representation. The paper is organized as follows: Section 2 gives properties of the conformable fractional derivative and methodology of solution. Section 3 gives the outline of the solution of Benney-Luke equation. In Section 4, we give the results and discussion. Section 5 gives the conclusion.

\section{The properties of the conformable fraction- al derivative and methodology of solution}

Some properties of the conformable fractional derivative is given using the following theorems:

Theorem 1: Let $\alpha \in(0,1]$ and suppose $u(t)$ and $v(t)$ are $\alpha$ differentiable at $t>0$. Then
a) $D_{t}^{\alpha}\left(t^{c}\right)=c t^{c-\alpha}$, for all $c \in \mathbb{R}$.
b) $\quad D_{t}^{\alpha}(a)=0$, for all constant function $u(t)=a$.
c) $\quad D_{t}^{\alpha}(a u(t))=a D_{t}^{\alpha}(u(t))$, for all $a$ constant.
d) $\quad D_{t}^{\alpha}(a u(t)+b v(t))=a D_{t}^{\alpha} u(t)+b D_{t}^{\alpha} v(t)$, for all $a, b \in \mathbb{R}$
e) $\quad D_{t}^{\alpha}(v(t) u(t))=D_{t}^{\alpha} v(t)(u(t))+u(t) D_{t}^{\alpha}(v(t))$. 
f) $\quad D_{t}^{\alpha}\left(\frac{u(t)}{v(t)}\right)=\frac{v(t) D_{t}^{\alpha} u(t)-u(t) D_{t}^{\alpha} v(t)}{v^{2}(t)}, v(t) \neq 0$.

g) If, in addition to $u(t)$ is differentiable, then $D_{t}^{\alpha} u(t)=t^{1-\alpha} \frac{d u}{d t}$.

Theroem 2: Let $\alpha \in(0,1]$ such $u(t)$ is differentiable and also $\alpha$ differentiable. Let $v(t)$ be a function defined in the range of $u(t)$ also differentiable, then

$D_{t}^{\alpha}(u(t) v(t))=t^{1-\alpha} v^{\prime}(t) u^{\prime}(v(t))$

See also [23-24]

Now, considering the following conformable fractional differential equation, we present the method:

$$
P\left(u, D_{t}^{\alpha_{1}} u, D_{x}^{\alpha_{2}} u, D_{t}^{\alpha_{1}} D_{x}^{\alpha_{2}} u, D_{t t}^{2 \alpha_{1}} u, D_{x x}^{2 \alpha_{2}} u, \ldots\right)=0, \quad 0<\alpha_{1}, \alpha_{2}<1 .
$$

By the wave transformation, we set

$$
u(x, t)=U(\xi), \xi=a \frac{x^{\alpha_{1}}}{\alpha_{1}}-b \frac{t^{\alpha_{2}}}{\alpha_{2}},
$$

where $\alpha_{1}, \alpha_{2}$ are fractional orders, $a$ and $b$ are nonzero constants. Substitution of transformation (5) into (4), we get a reduced ordinary differential equation of the polynomial form

$$
Q\left(U(\xi), \frac{d}{d \xi} U(\xi), \frac{d^{2}}{d \xi^{2}} U(\xi), \ldots\right)=0
$$

\section{Solution of conformable space-time Benney- Luke equation}

Consider the conformable space-time Benney-Luke equation

$$
D_{t t}^{2 \alpha} u-D_{x x}^{2 \alpha} u+p D_{x x x x}^{4 \alpha} u-q D_{x x t}^{4 \alpha} u+3 D_{x x t}^{3 \alpha} u^{2}=0, \alpha \in(0,1] .
$$

On using the transformation Eq. (5), we get a reduced ordinary differential equation as follows

$b^{2} u^{\prime \prime}-a^{2} u^{\prime \prime}+a^{4} p u^{\prime \prime \prime \prime}-q a^{2} b^{2} u^{\prime \prime \prime \prime}-3 a^{2} b\left(u^{2}\right)^{\prime \prime \prime}=0$.

Balancing the highest order derivative with the highest nonlinear order in Eq. (8) after integrating trice with zero constant of integration assumption,we get

$N=1$.

\subsection{Soliton solution by Kudryashov method}

From Eq. (9), Kudryashov method offers a truncated series solution of the form:

$U(\xi)=a_{0}+a_{1} \Phi(\xi)$

Where, $a_{0}$ and $a_{1}$ are constants and $\Phi(\xi)$ is given by the function

$$
\Phi(\xi)=\frac{1}{1+d \exp (\xi)},
$$

Which satisfies the differential equation

$$
\Phi^{\prime}(\xi)=\Phi^{2}(\xi)-\Phi(\xi)
$$

Thus, substituting Eq. (10) with its necessary derivatives alongside Eq. (12) into Eq. (8); equating the coefficients of $\Phi(\xi)$ to zero we get the following algebraic equations with the help of Mathematica software:

$-a^{2} a_{1}+b^{2} a_{1}+a^{4} p a_{1}-a^{2} b^{2} q a_{1}+6 a^{2} b a_{0} a_{1}=0$,

$3 a^{2} a_{1}-3 b^{2} a_{1}-15 a^{4} p a_{1}+15 a^{2} b^{2} q a_{1}-42 a^{2} b a_{0} a_{1}+24 a^{2} b a_{1}^{2}=0$,

$-2 a^{2} a_{1}+2 b^{2} a_{1}+50 a^{4} p a_{1}-50 a^{2} b^{2} q a_{1}+72 a^{2} b a_{0} a_{1}-114 a^{2} b a_{1}^{2}=0$,

$-60 a^{4} p a_{1}+60 a^{2} b^{2} q a_{1}-36 a^{2} b a_{0} a_{1}+162 a^{2} b a_{1}^{2}=0$,

$24 a^{4} p a_{1}-24 a^{2} b^{2} q a_{1}-72 a^{2} b a_{1}^{2}=0$.

Solving the above system, we get the following:

Case 1:

$a_{0}=a_{0}, \quad a_{1}=-2 a_{0}, \quad a= \pm \frac{6 a_{0}}{\sqrt{p^{2}-2 p q+q^{2}}}, \quad b=-\frac{6 a_{0}}{p-q}$

Which produces

$u_{1,2}(x, t)=a_{0}-\frac{2 a_{0}}{1+d \exp \left( \pm \frac{6 a_{0}}{\sqrt{p^{2}-2 p q+q^{2}}} \frac{x^{\alpha}}{\alpha}+\frac{6 a_{0}}{p-q} \frac{t^{\alpha}}{\alpha}\right)}$,

(see Fig. 1).

\subsection{Soliton solution by modified extended $\tanh$ method}

From Eq. (9), modified extended tanh method offers a truncated series solution of the form:

$U(\xi)=a_{0}+a_{1} \Phi(\xi)+\frac{b_{1}}{\Phi(\xi)}$,

Where, $A_{0}, A_{1}$ and $B_{1}$ are constants and $\Phi(\xi)$ is given by the function

$\Phi(\xi)=\tanh \xi$

Which satisfies the differential equation

$\Phi^{\prime}(\xi)=r+\Phi^{2}(\xi)$

Where $r$ is constant. Also, Eq. (16) has the following structure of solutions:

a) If $r<0$, then

$\Phi(\xi)=-\sqrt{-r} \tanh (\sqrt{-r} \xi)$,

$\Phi(\xi)=-\sqrt{-r} \operatorname{coth}(\sqrt{-r} \xi)$,

b) If $r=0$, then

$\Phi(\xi)=-\frac{1}{\xi}$

c) If $r>0$, then

$\Phi(\xi)=\sqrt{r} \tan (\sqrt{r} \xi)$,

$\Phi(\xi)=-\sqrt{r} \cot (\sqrt{r} \xi)$. 
Therefore, substituting Eq. (14) with its necessary derivatives alongside Eq. (16) into Eq. (8); equating the coefficients of $\Phi(\xi)$ to zero we get the following algebraic equations with the help of Mathematica software:

$24 a^{4} p r^{4} b_{1}-24 a^{2} b^{2} q r^{4} b_{1}+72 a^{2} b r^{3} b_{1}^{2}=0,36 a^{2} b r^{3} a_{0} b_{1}=0$

$-2 a^{2} r^{2} b_{1}+2 b^{2} r^{2} b_{1}+40 a^{4} p r^{3} b_{1}-40 a^{2} b^{2} q r^{3} b_{1}+120 a^{2} b r^{2} b_{1}^{2}=0$,

$-2 a^{2} b_{1}+2 b^{2} b_{1}+16 a^{4} p r^{2} b_{1}-16 a^{2} b^{2} q r^{2} b_{1}+48 a^{2} b r b_{1}^{2}=0$,

$-12 a^{2} b r^{2} a_{0} a_{1}+12 a^{2} b r a_{0} b_{1}=0,48 a^{2} b r^{2} a_{0} b_{1}=0,-48 a^{2} b r a_{0} a_{1}=0$,

$-2 a^{2} r a_{1}+2 b^{2} r a_{1}+16 a^{4} p r^{2} a_{1}-16 a^{2} b^{2} q r^{2} a_{1}-48 a^{2} b r^{2} a_{1}^{2}=0$,

$-2 a^{2} a_{1}+2 b^{2} a_{1}+40 a^{4} p r a_{1}-40 a^{2} b^{2} q r a_{1}-120 a^{2} b r a_{1}^{2}=0$

$-36 a^{2} b a_{0} a_{1}=0,24 a^{4} p a_{1}-24 a^{2} b^{2} q a_{1}-72 a^{2} b a_{1}^{2}=0$

Solving the above system, we get the following:

Case 1:

$a_{0}=0, \quad a_{1}=a_{1}, \quad b_{1}=0$,

$a= \pm \frac{3 a_{1}}{\sqrt{p^{2}-2 p q+q^{2}}}, \quad b=\frac{3 a_{1}}{p-q}, \quad r=r$.

Which produces

$u_{1,2}(x, t)=-a_{1} \sqrt{-r} \tanh \left(\sqrt{-r}\left(\begin{array}{l} \pm \frac{3 a_{1}}{\sqrt{p^{2}-2 p q+q^{2}}} \frac{x^{\alpha}}{\alpha} \\ -\frac{3 a_{1}}{p-q} \frac{t^{\alpha}}{\alpha}\end{array}\right)\right), r<0$,

(See Fig. 2).

$u_{3,4}(x, t)=-a_{1} \sqrt{-r} \operatorname{coth}\left(\sqrt{-r}\left(\begin{array}{l} \pm \frac{3 a_{1}}{\sqrt{p^{2}-2 p q+q^{2}}} \frac{x^{\alpha}}{\alpha} \\ -\frac{3 a_{1}}{p-q} \frac{t^{\alpha}}{\alpha}\end{array}\right)\right), r<0$,

(See Fig. 3).

$u_{5,6}(x, t)=a_{1} \sqrt{r} \tan \left(\sqrt{r}\left(\begin{array}{l} \pm \frac{3 a_{1}}{\sqrt{p^{2}-2 p q+q^{2}}} \frac{x^{\alpha}}{\alpha} \\ -\frac{3 a_{1}}{p-q} \frac{t^{\alpha}}{\alpha}\end{array}\right)\right), r>0$,

(See Fig. 4)

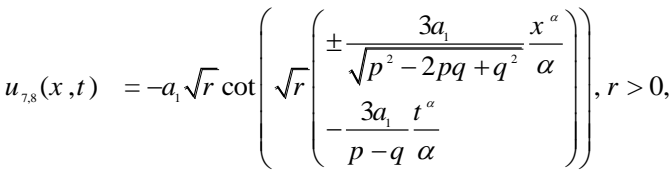

$a_{0}=0, \quad a_{1}=a_{1}, \quad b_{1}=0, \quad a= \pm \frac{3 a_{1}}{\sqrt{p^{2}-2 p q+q^{2}}}$

$b=\frac{3 a_{1}}{p-q}, \quad r=0$.

Which produces

$u_{9,10}(x, t)=\frac{a_{1}}{\frac{3 a_{1}}{\sqrt{p^{2}-2 p q+q^{2}}} \frac{x^{\alpha}}{\alpha}+\frac{3 a_{1}}{p-q} \frac{t^{\alpha}}{\alpha}}$

(See Fig. 6)

Case 3:

$a_{0}=0, a_{1}=a_{1}, b_{1}=b_{1}, \quad a= \pm \frac{3 a_{1}}{\sqrt{p^{2}-2 p q+q^{2}}}$,

$b=\frac{3 a_{1}}{p-q}, \quad r=-\frac{9 a_{1} b_{1}}{a^{2}(p-q)^{2}},<0$.

Which produces

$$
\begin{aligned}
& u_{11,12}(x, t)=-a_{1} \sqrt{\frac{9 a_{1} b_{1}}{a^{2}(p-q)^{2}}} \tanh \left(\sqrt{\frac{9 a_{1} b_{1}}{a^{2}(p-q)^{2}}} \xi\right) \\
&-\frac{b_{1}}{\sqrt{\frac{9 a_{1} b_{1}}{a^{2}(p-q)^{2}}}} \operatorname{coth}\left(\sqrt{\frac{9 a_{1} b_{1}}{a^{2}(p-q)^{2}}} \xi\right), \\
& \xi=\quad \pm \frac{3 a_{1}}{\sqrt{p^{2}-2 p q+q^{2}}} \frac{x^{\alpha}}{\alpha}-\frac{3 a_{1}}{p-q} \frac{t^{\alpha}}{\alpha} .
\end{aligned}
$$

(See Fig. 7)

\section{Graphical representations}

In the present section, we give the graphical representations of the conformable space-time fractional Benney-Luke equation'ssolutions obtained and presented in Eq. (13) and Eqs. (17-22). The graphs are ploted for $0 \leq \mathrm{x} \leq 2$ and $0 \leq \mathrm{t} \leq 1$ and the used papameters are prescribed below each figure given in Fig1-7 as follows:

(See Fig. 5)

Case 2: 


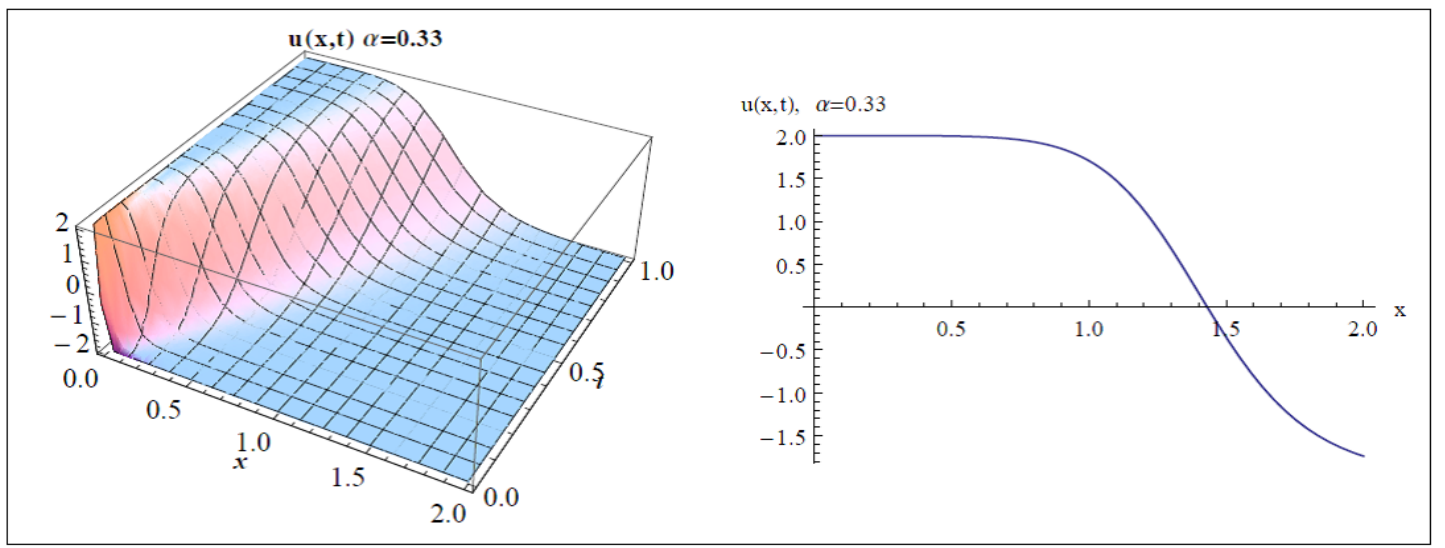

Fig. 1: Profiles of Eq. (13), Subsituting the Values of the Parameters $p=2 ; q=0.2, a_{0}=2 ; d=0.7 ; \alpha=0.33$. and $t=1$ for the 2D Graphs.

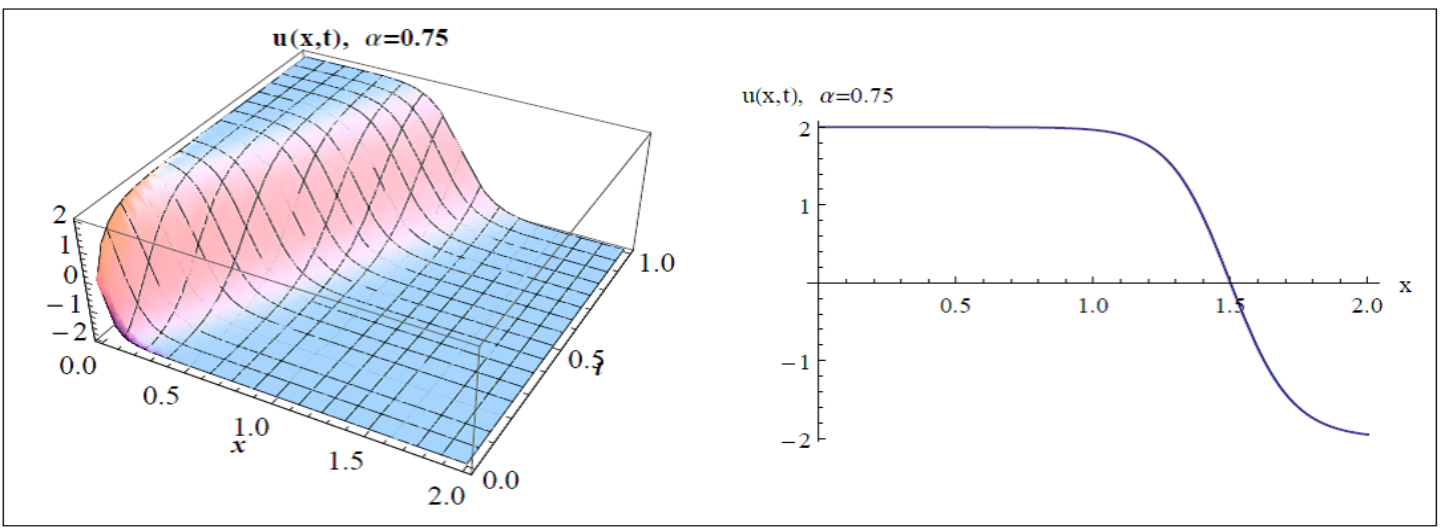

Fig. 2: Profiles of Eq. (17), Subsituting the Values of the Parameters $a_{1}=2, p=1.8, q=0.6, r=-1, \alpha=0.75$ and $t=1.5$ for the 2D Graph.

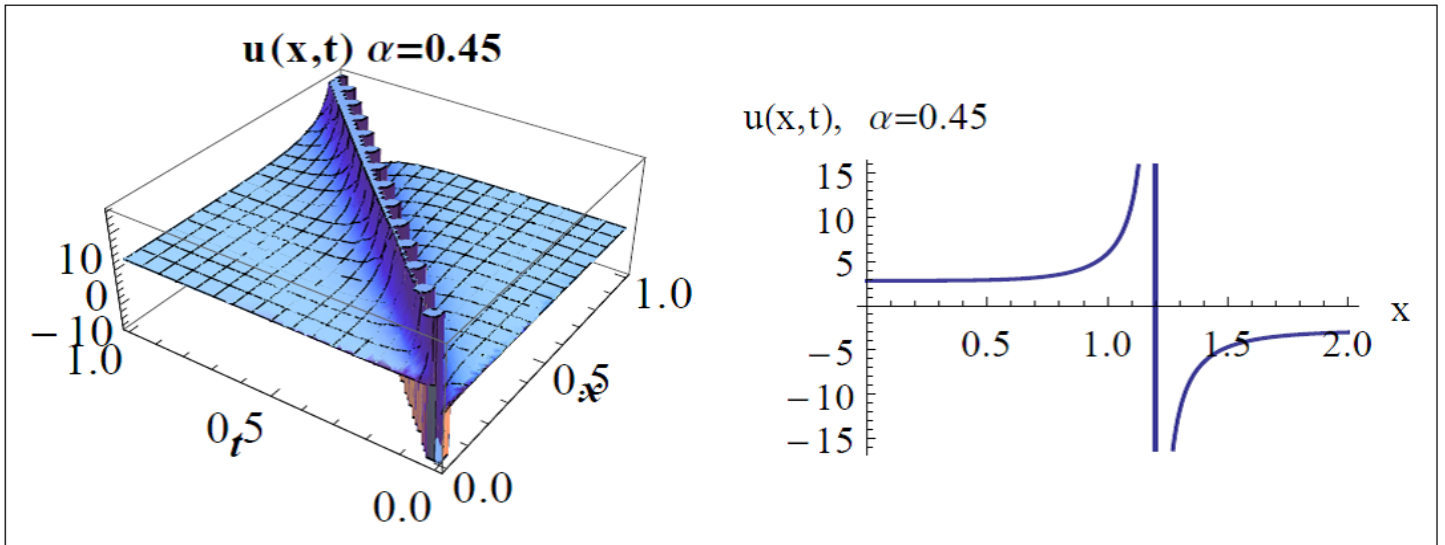

Fig. 3: Profiles of Eq. (18), Subsituting the Values of the Parameters $a_{1}=2, p=2, q=1, r=-4, \alpha=0.45$ and $t=1$ for 2D Graph.

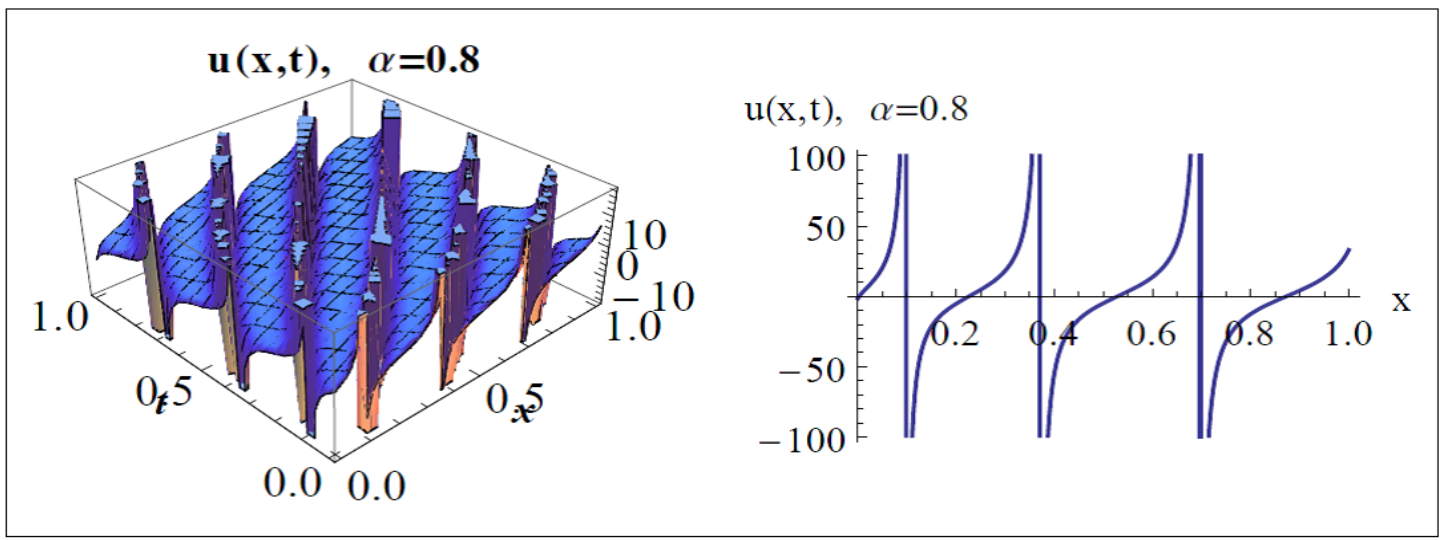

Fig. 4: Profiles of Eq. (19), Subsituting the Values of the Parameters $a_{1}=2, p=2, q=1, r=2, \alpha=0.8$ and $t=2.5$ for the 2D Graph. 


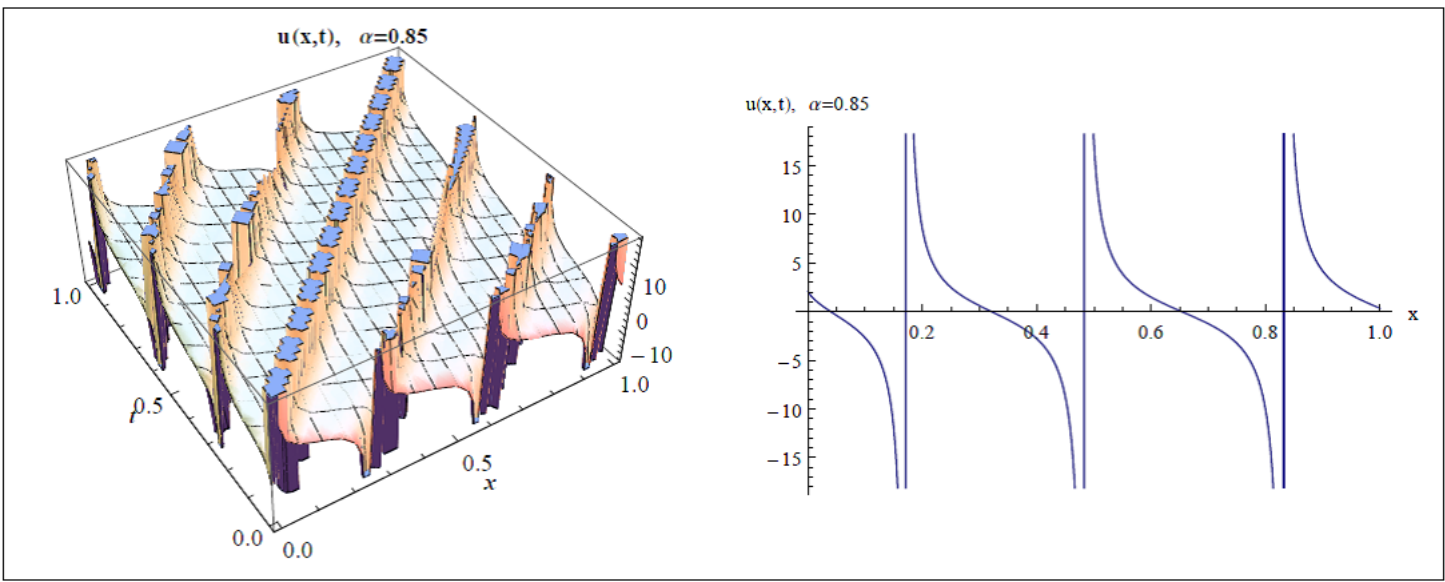

Fig. 5: Profiles of Eq. (20), Subsituting the Values of the Parameters $a_{1}=2.3, p=2, q=1, r=1.5, \alpha=0.85$ and $t=2$ for the 2D Graph.

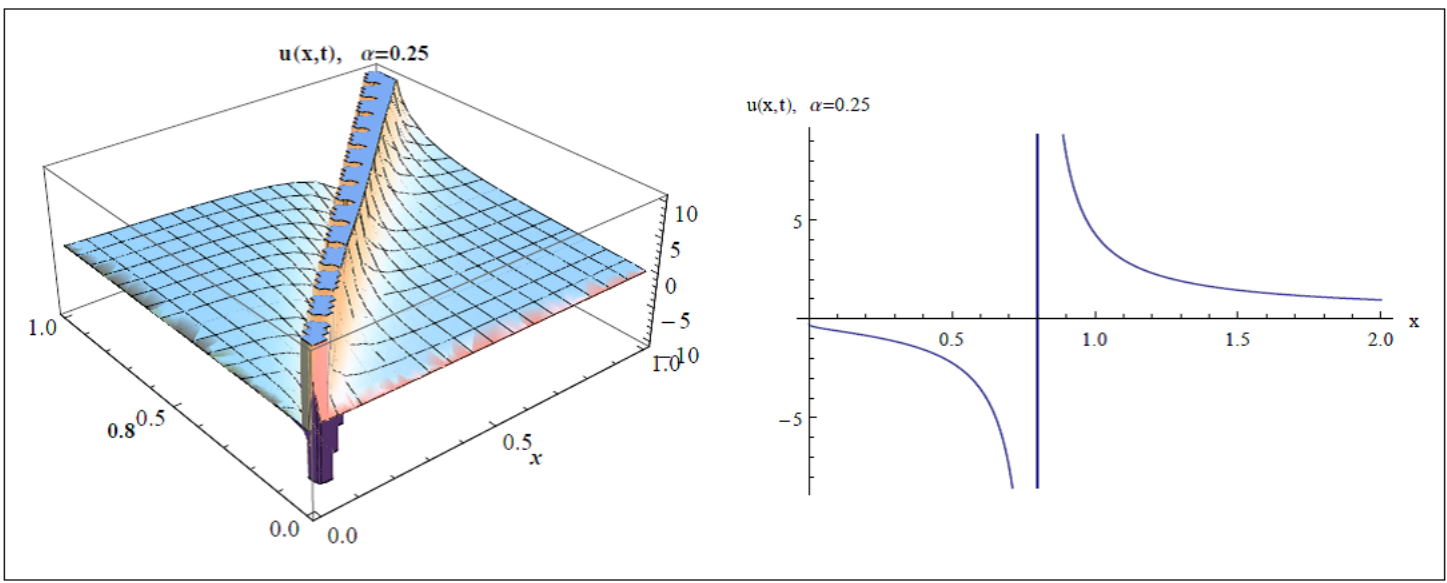

Fig. 6: Profiles of Eq. (21), Subsituting the Values of the Parameters $a_{1}=2.8, p=3, q=1, r=0, \alpha=0.25$ and $t=0.25$ for 2D Graph.

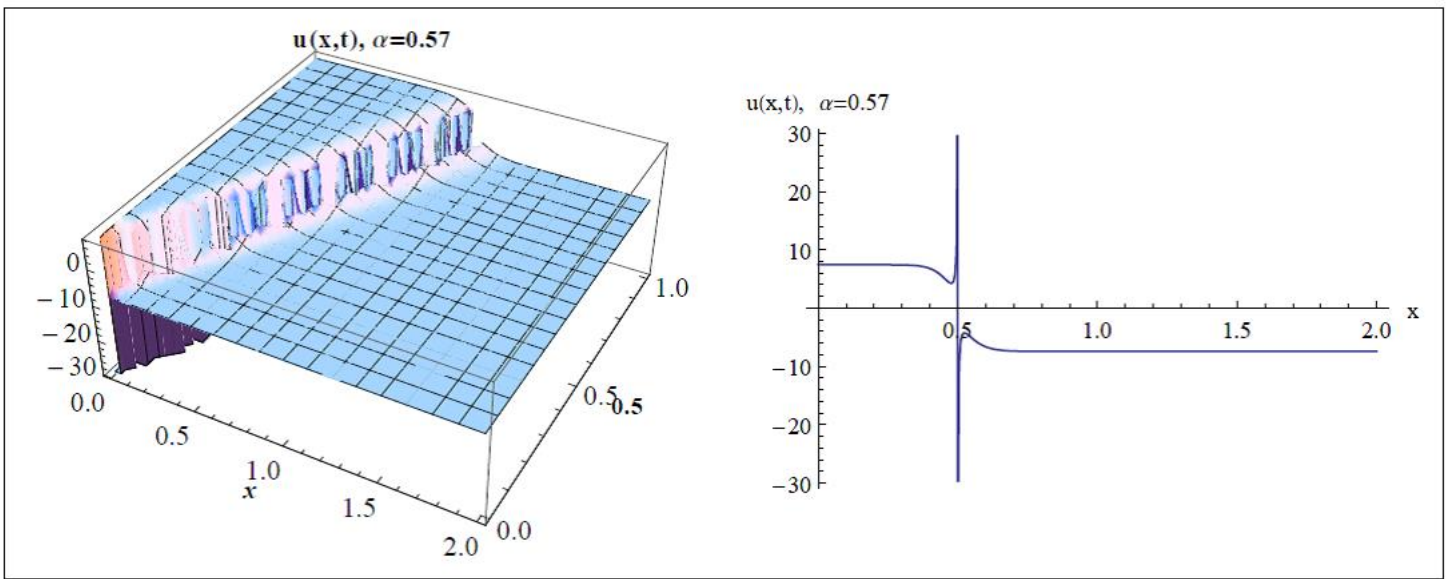

Fig. 7: Profiles of Eq. (22), Subsituting the Values of the Parameters $a_{1}=1.8, b_{1}=1.2, p=2, q=1, a=1.2, \alpha=0.57$ and $t=2.4$ for the 2D Graph.

\section{Results and discussion}

This study effectively examines and constructs varieties of solutions for the conformable space-time fractional Benney-Luke equation consisting of exponential, trigonometric and hyperbolic function using two reliable methods of Kudryashov and modified extended than expansion methods, respectively. Various graphical illustrations are depicted using the Mathematica software ranging from Fig. 1 through Fig. 7 at various chosen values of $\alpha$. different solitons solutions such the singular periodic wave's shapes, kinktype solution shapes and singular soliton solution shapes are obtained for the problem. Finally, it is worth mentioning that at $\alpha=1$, the classical Benney-Luke equation is recovered, which shows that clearly these reliable methods would give some of the exact analytical solutions obtained in [3].

\section{Conclusion}

In conclusion, the conformable space-time fractional BenneyLuke equation is extensively examined in this study by employing two reliable analytical methods. The first method was the powerful Kudryashov method that gives exponential function solutions while the second method was the modified extended than expansion method with the Riccati differential equation and yields different trigonometric and hyperbolic function solutions, respectively. Thus, the method can be used in treating various 
nonlinear space-time fractional differential equations. Finally, the graphs of all solutions are depicted for visualization.

\section{References}

[1] Wazwaz A. (2017). A variety of soliton solutions for the Boussinesq-Burgers equation and the higher-order BoussinesqBurgers equation. Filomat. 31(3) 831-840.

[2] Pego RL., Quintero J.R. (1999). Two dimensional solitary waves for Benney-Luke equation. Physica D.132:476-496 https://doi.org/10.1016/S0167-2789(99)00058-5.

[3] Islam S.M.R., Khan K., Al Woadud K.M.A. (2017). Anatytica studies on the Benney-Luke equation in mathematical physics. Waves in Random Media and Complex Media.

[4] Kudryashov N.A. (2012). One method for finding exact solutions of nonlinear differential equations. Communication in Nonlinear Science and Numerical Simulation.17(6) 2248-2253. https://doi.org/10.1016/j.cnsns.2011.10.016.

[5] Raslan K.R., Khalid K.A., Shallal M.A. (2017). The modified extended tanh method with the Riccati equation for solving the space-time fractional EW and MEW equations. Chaos, Solitons Fractals.103404-409. https://doi.org/10.1016/i.chaos.2017.06.029.

[6] Raslan K.R., Talaat S.E., Khalid K.A. (2017).Exact solution of space-time fractional coupled EW and coupled MEW equations.The EuropeanPhysical Journal Plus.132: 319. https://doi.org/10.1140/epip/i2017-11590-9.

[7] Hosseini K., Ayati Z., Ansari R. (2017).New exact solutions of the Tzitzéica type equations arising in nonlinear optics using a modified version of the improved $\tan (\varphi(\xi) / 2)$-expansion method. Opt. Quantum Electron.49: 273. https://doi.org/10.1007/s11082-0171094-4.

[8] Nuruddeen R.I., Nass A.M. (2017).Exact solutions of wave-type equations by the Aboodh decomposition method. Stochastic Modeling and Applications . 21(1): 23-30.

[9] Raslan K.R., Talaat S.E., Khalid K.A. (2017). New exact solution of coupled general equal width wave equation using sine-cosine function method.Journal of the Egyptian Mathematical Society.25 350-354. https://doi.org/10.1016/j.joems.2017.03.004.

[10] Alquran M., Jaradat H.M., Syam M.I. (2017).Analytical solution of the time-fractional Phi-4 equation using modified power series method. Nonlinear Dynamics.

[11] Yaslan H.C. (2017).New Analytic solutions of the conformable space-time fractional Kawahara equation. Int J. light Elect Opt. https://doi.org/10.1016/j.ijleo.2017.04.015.

[12] Zhang Y., Feng Q. (2013). Fractional Riccati equation rational expansion method for fractional differential equations. Applied Mathematics \& Information Science. 7(4) 1575-1584. https://doi.org/10.12785/amis/070443.

[13] Cenesiz Y., Baleanu D., Kurt A., Tasbozan O. (2016). New exact solutions of Burgers' type equations with conformable derivative. Waves in Random Media and Complex Media.

[14] Hawlader F., Kumar D. (2017). A variety of exact analytical solutions of extended shollow water wave equations via improved $\left(\mathrm{G}^{\prime} / \mathrm{G}\right)$-expansion method. International Journal of Physical Research. 5(1) 21-27. https://doi.org/10.14419/ijpr.v5i1.7429.

[15] Sivaporn A., Surattana S., Sanoe K. (2017). New exact solutions for the time fractional clannish random walker's parabolic equation by the improved $\tan (\varphi(\xi) / 2)$-expansion method. AMM 2017. Chiang Mai University, Chiang Mai, Thailand.

[16] Nuruddeen R.I. (2017). Elzaki decomposition method and its applications in solving linear and nonlinear Schrodinger equations.Sohag Journal of Mathematics. 4(2) 1-5.

[17] Inc M., Yusuf A., Aliyu A.I. (2017). Dark optical and other soliton solutions for the three different nonlinear schrodinger equations.Optical and Quantum,49(11)

[18] Inc M., Yusuf A.,Aliyu A.I., Baleanu D. (2017). Optical soliton solutions for the higher-order dispersive cubic-quintic nonlinear Schrodinger equation.Superlattices and Microstructures.https://doi.org/10.1016/j.spmi.2017.08.059.

[19] Baskonus H.M., Sulaiman T.A., Bulut H. (2017). New solitary wave solutions to the $(2+1)$-dimensionalCalogero-BogoyavlenskiiSchiff and the Kadomtsev-Petviashvilihierarchy equations. Indian J Phys. https://doi.org/10.1007/s12648-017-1033-z.

[20] Bulut H., Sulaiman T.A., Baskonus H.M., Erdogan F. (2017). On the newhyperbolic and trigonometric structuresto the simplified-
MCHand SRLWequations. The European Physical Journal Plus. 132: 350

[21] Kilbas AA, Srivastava HM, Trujillo JJ. (2006). Theory and Applications of Fractional Differential Equations. Elsevier, Amsterdam

[22] Debnath L. (2003). Recent applications of fractional calculus to science and engineering. Inernational Journal of Mathematics and Mathematical Sciences.54 3413-3442. https://doi.org/10.1155/S161171203301486.

[23] Khalil R. et al. (2014).A new definition of fractional derivative, $J$. Comput. Appl. Math.264 65-701. https://doi.org/10.1016/j.cam.2014.01.002.

[24] Abu Hammad M., Khalil R. (2014). Conformable fractional heat differential equation, Int. J. Pure Appl. Math. 94 (2) 215-221. https://doi.org/10.12732/ijpam.v94i2.8.

[25] Atangana A., Baleanu D. (2016) New fractional derivatives with nonlocal and non-singular kernel: theory and application to heat transfer model. Therm Scie.arXiv preprint arXiv: 1602.03408. https://doi.org/10.2298/TSCI160111018A. 